\title{
Filament Activity Associated With an Umbral Flare
}

\author{
Wei Li and Jingxiu Wang \\ Beijing Astronomical Observatory, Chinese Academy of Sciences \\ Beijing 100080, China
}

\begin{abstract}
An umbral flare in NOAA 6223 was observed at Huairou Solar Observing Station of Beijing Astronomical Observatory. Vigorous filament activity took place in association with this umbral flare. Several filaments formed and/or disappeared, leaving the magnetic neutral lines pointing to or rooted in the $P$ spot in which the umbral flare occurred. We describe the observed configuration and evolution of the vector magnetic fields in the photosphere, and Doppler velocity patterns in the chromosphere during the course of the umbral flare and related filament activity.
\end{abstract}

\section{Introduction}

In most case flare patches are in plages. They usually avoid sunspots and, even when they expand, they prefer the spot-free space. Often the flare's expansion stops at the boundary of a large spot. Some large flares do penetrate into the umbra of a spot or spot group. The umbral flare named by Tang (1978) differs from the above-mentioned flares in that the umbra flare patch is confined to the umbra; more importantly, it originates in the umbra. It is not the result of the spreading or expansion of the flare patch. In a survey of many years of $\mathrm{H}_{\beta}$ movies, only five umbral flares were found (Tang 1978). Since then, two flares which were characterized by umbral flares in the $\mathrm{H}_{\beta}$ chromospheric line were observed in active region (AR) 6223 at Huairou Solar Observing Station (HSOS) on August 23, 1990. One of the two flares was a 1N/C3.9 flare, and the other a microflare. The dominant $\mathrm{P}$ spot with no bridge in the umbra nor gap in the penumbra has north polarity. The $\mathrm{F}$ spot is very tiny and has south polarity. In this paper, we shall describe for the first time the observed configuration and evolution of the vector magnetic fields in the photosphere, and Doppler velocity patterns in the chromosphere in the course of the $1 \mathrm{~N} / \mathrm{C} 3.9 \mathrm{umbral}$ flare and related filament activity.

\section{Filament Activity}

Figure 1 shows the filtergrams of the active region in $\mathrm{H}_{\beta}$ chromospheric line. At least six filaments existed within the active region before and during the flares. They pointed to or were rooted in the $\mathrm{P}$ spot, and formed and/or disappeared before the flare. We could easily see the filament 2,5 and dimly see the filament 1 and 4 at 00:54 UT. Later, the filament 2, 4 became darker and thicker and filament 3 appeared, a part of the filament 5 broke, and the position and shape 
of the filament 1 changed a little at 03:21 UT. Nevertheless, all of the filaments still were rooted in the umbra of the P spot at 03:21 UT, except the filament 3. It is interesting that a segment of the filament 1 became darker and thicker and another segment of the filament 1 became bright at this time. At 04:41 UT, the filament 2 and 1 were broken, and 3 and 5 disappeared. At 06:53 $\mathrm{UT}$, the filament 1 with a brightening feature appeared again, and filament 2 almost completely disappeared. At 07:30 UT, the filament 2 appeared again and the umbral flare occurred. A segment of the filament 4 became brighter and broken at 07:39 UT. After the flare the filaments 2 and 5 appeared again. Table 1 summarizes the time sequence of filament activities in association with the umbral flares.

Table 1, Temporal Relationship Between Flares and Filaments

\begin{tabular}{cccc}
\hline \hline Filament & Appearance & Activation & Disappearance \\
\hline 1 & $00: 54$ & $03: 21,04: 41,06: 53$ & \\
2 & $00: 54,07: 30,08: 16$ & $03: 21,04: 41$ & $06: 53,07: 33$ \\
3 & $03: 21$ & & $04: 41$ \\
4 & $00: 54$ & $03: 21,07: 30,07: 39$ & \\
5 & $00: 54$ & $03: 21$ & $04: 41$ \\
Flare & $07: 30$ & maximum at $07: 39$ & $08: 16$ \\
\hline
\end{tabular}

\section{Magnetic Field and Velocity Field}

Figure 2 shows the vector magnetograms of the active region, and the positions of the flare and the filaments. We can see that both of the flare ribbons 1 and the knotted-like ribbon 2 were far from the neutral line. There was only north polarity longitudinal field and 750-1250 G transverse field in the photosphere underlying a large segment of the filament $1,2,4$, and 5 which was directly rooted in the umbra of the $\mathrm{P}$ spot. However, the other foot of the filament 1 , 2 and 4 was located in the polarity inversion zone. In the filament's vicinity, the main sites of new emergent flux and flux cancellations are marked by a square and 'A'. At 00:31 UT, N polarity dominated in the square and, later, S polarity flux emerged and grew quickly in area and in strength. By 07:23 UT, S polarity has already dominated the square in the vicinity of the filament and in the filament channel. We also found that flux cancellation around the mark ' $\mathrm{A}$ ' in Figure 2 occurred during the period 00:31 to 07:23 UT. We found that the strength of the transverse field in the area of the $p$ spot did not change, but the alignments obviously changed during the period 23:26 to 07:13 UT. Specifically, the alignments of the transverse fields in the north half part of the $p$ spot changed counterclockwise and in the south half part of the $p$ spot changed clockwise.

It seems that the filament $1,2,4$ and 5 could be divided into two segments along the direction of the filament axis, and the red-shifted feature (downflow) in a large segment of the filament was observed pointing to or rooted in the $p$ spot. The blue-shifted feature in the remaining parts of the filament, especially, in the middle part of the filament, was observed before the flare. At 03:21 UT, a segment of the filament 1 brightened, and at 06:53 UT the full filament 1 brightened again. Therefore, as a result the Doppler velocity pattern showed concentrated red-shift globally, i.e., material was flowing into the P spot along 

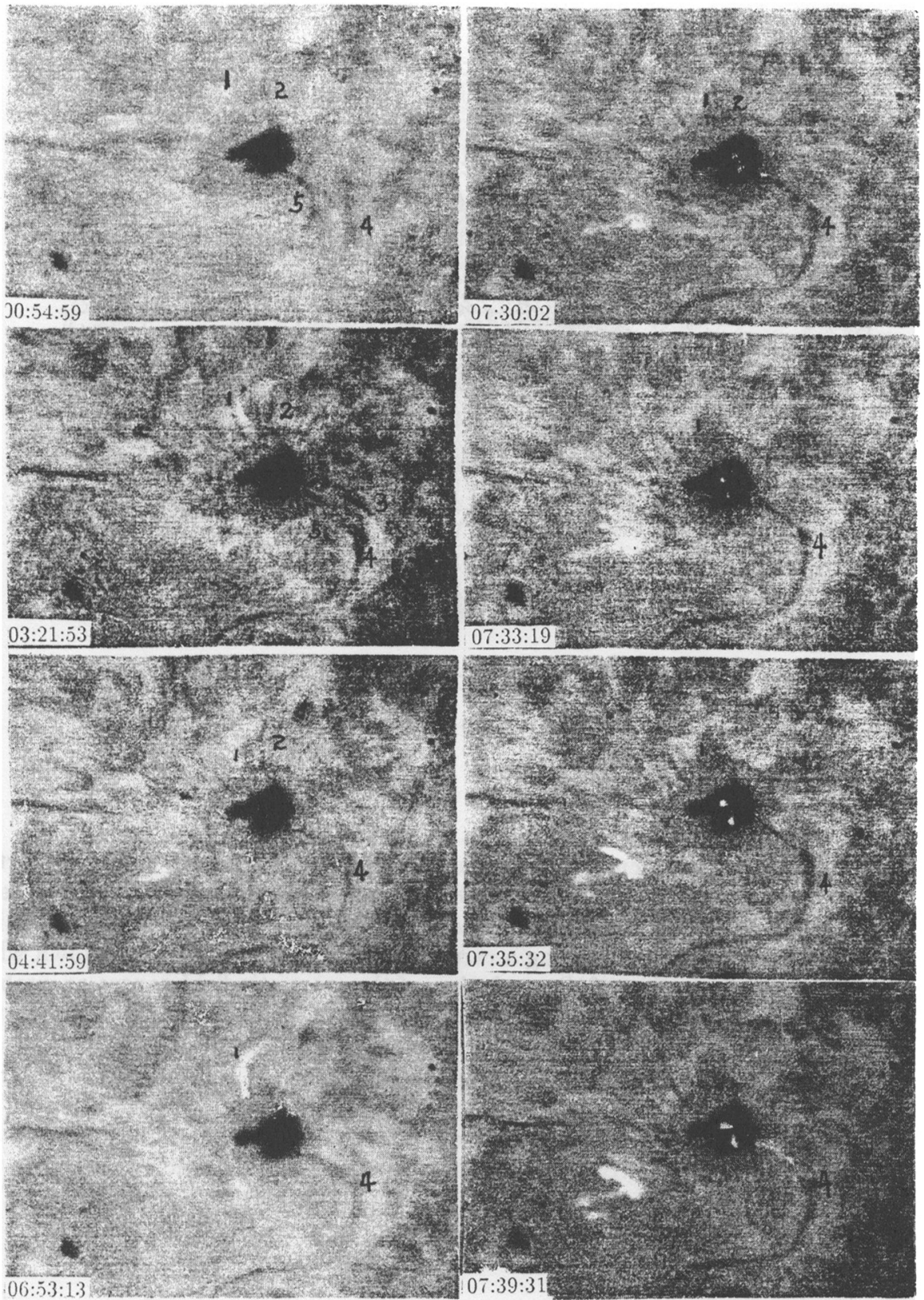

Figure 1. The filtergrams of the active region $A R 6223$ in the $H \beta$ chromospheric line. 


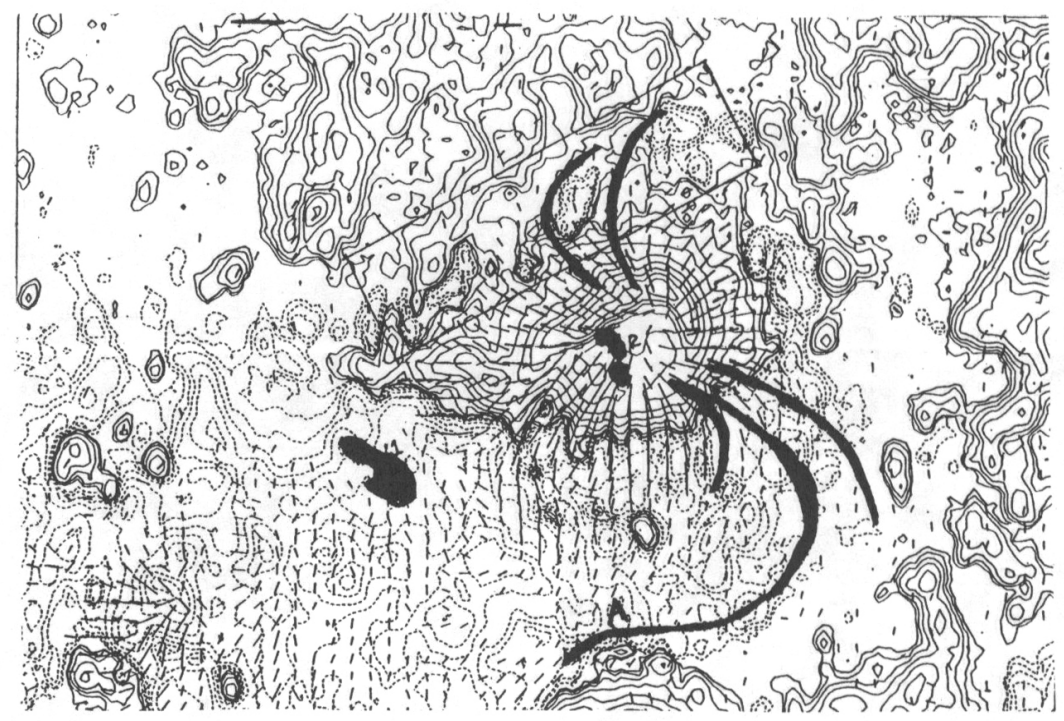

Figure 2. The vector magnetogram of the active region AR 6223.

the filaments. At 07:30 UT, the umbral flare occurred.

\section{Summary}

The fact that a flare occurred in the umbra indicates that energy was transported downward along the field lines which were longer than those close to the magnetic neutral line, or that the magnetic field was of a very complicated configuration in the higher atmosphere. Being a rare phenomenon, it is totally unknown what particular configuration of the evolution of the magnetic field causes an umbral flare. However, it has been shown that vigorous filament activity is associated with the umbral flares. Those filaments have one foot rooted in the main $\mathbf{P}$ sunspot (McIntosh 1972), while the other outer foot is located in the polarity inversion zones where there is continuous flux emergence and cancellation. We speculate that at the outer feet of the filaments the magnetic lines of force are opened by magnetic reconnection. High energy particles precipitate along the opened field lines into the umbral atmosphere, causing the umbral flares. This scenario might explain the observed field evolution and velocity pattern.

\section{References}

McIntosh, P. S. 1972, Rev. Geophys. Space Phys., 10, 837

Tang, F. 1987, Sol. Phys., 60, 119 